\section{Never underestimate renal colic: a case of pelvic rupture}

\author{
Tiziana Battista, ${ }^{1}$ Domenico Cantiello, ${ }^{2}$ \\ Marta Salzillo, ${ }^{2}$ Ermenegildo Pezzulo, ${ }^{2}$ \\ Diego Paternosto ${ }^{2}$
}

'School of Emergency and Urgency

Medicine, Second University of Naples, Naples; ${ }^{2}$ Emergency Department, S. Anna

and S. Sebastiano Hospital, Caserta, Italy

\section{Abstract}

Authors describe a case of pelvic rupture as a consequence of renal colic. Pelvic rupture is an unusual condition that often is not considered in patients suffering renal colic. In this case diagnosis was not considered on ultrasonography, it was suspected when acute abdominal symptoms began and it was confirmed by computed tomography.

\section{Introduction}

Spontaneous rupture of the renal pelvis with urine extravasation into peripelvic and retroperitoneal spaces is not a common pathologic event. Symptoms may be various, going from mild diffuse pain, typical renal colic to acute abdomen, so that diagnosis is often delayed. It is due, in most cases, to an ureteral obstruction by calculus; other causes are trauma, tumors, pregnancy, diagnostic and interventional procedures. ${ }^{1-4}$ Diagnosis, as reported in literature, is often difficult and has to be differentiated with numerous causes of abdominal pain due to involvement of perirenal abdominal structures and organs. It is usually recognized with a contrast-enhanced computed tomography (CT) showing passage of contrast medium into peripelvic, perirenal and retroperitoneal spaces. ${ }^{5}$ Few cases are reported in literature $\mathrm{e}^{6-9}$ and we report our experience of spontaneous rupture of the renal pelvis in a case associated with ureteral calculus. Generally, ureteral stones are eliminated with great pain but without complications; sometimes they may be associated with complications as hydronephrosis, impaired renal function, urinary tract infections. Ulceration or clear rupture constitute unusual complications. Urine extravasion in obstructive calculosis may be due to sweating fornices caused by a sudden increase of intrapielic pressure (critical levels reported between 25 and $75 \mathrm{~mm} \mathrm{Hg}$ ) worsened by coughing and vomiting, or a frank rupture caused by a pelvic or ureteral lesion.

\section{Case Report}

A 61-year-old man presented in our Emergency Room (ER) complaining of diffuse abdominal pain. Two days previously he had suffered from a sudden severe typical renal colic pain with vomiting and sweating.

In his past history he had been affected by hypertension, gastroesophageal reflux disease, right renal calculuses treated with a temporary stent implantation because the location of stones did not allow him to be treated with extracorporeal ultrasounds.

Renal colic, immediately recognized by the patient, was treated at home with intramuscular administration of diclofenac and subsequently, due the resumption of pain, with some intravenous infusions of spasmolytic, ketorolac, ranitidine at home. The next day, the pain disappeared and a renal echo was performed showing no pelvic dilatation nor reno-ureteral stones. However, in the evening, diffuse abdominal pain began with difficulties to get rid of faeces and gas. Moreover, poor diuresis was noted in spite of much liquid being administered. Next morning, the patient enters our ER. Physical examination reveals an alert and collaborating patient with stable vital parameters, tight but handling abdomen, alvo closed for faeces and gas, rectal digital exploration negative for fecal impaction; an abdominal echo is performed showing no hydronephrosis but showing a little liquid collected in his abdomen; direct abdomen Rx shows diverticulosis without air-fluid levels. Laboratory analysis shows high levels of creatinine (1.9 $\mathrm{mg} / \mathrm{dL}$ ), ESV, PCR, procalcitonin, fibrinogen.

Diverticula inflammation is one of the pathologic conditions that, because of its kind of symptoms, may mime renal calculi, and so our patient begins therapy with Metronidazole. ${ }^{10} \mathrm{~A}$ few minutes after therapy is given, the patient begins to suffer from shaking shivers with a sudden increase of body temperature in a few minutes (CT: $38,8^{\circ} \mathrm{C}$ ). Immediately a contrast-enhanced CT scan is performed showing contrast leakage in perirenal and retroperitoneal spaces because of an ulceration of the renal pelvis (Figure 1), and the presence of a radiopaque ureteral stone about $7 \mathrm{~mm}$ large.

Because of sudden evolution of symptoms, immediately a double $\mathbf{J}$ ureteral stent is delivered and antibiotic therapy with piperacillin and tazobactam i.v. is prescribed: so there is a prompt resolution of symptoms. Also, laboratory exams, after peaking on the second day, quickly normalize. After a week on antibiotic infusional therapy, a CT-scan is performed to verify a good stent position (Figure 2) no trace of the stone is noted and the patient is discharged and advised to continue oral antibiotic therapy with ciprofloxacin for seven days. After
Correspondence: Tiziana Battista, School of Emergency and Urgency Medicine, Second University of Naples, via Montelungo 21, 81100 Caserta, Italy.

Tel: +39.320 .6188356 .

E-mail: tizyb85@gmail.com

Key words: Pelvic rupture; Renal colic; Ultrasounds; Computed tomography.

Conflict of interest: the authors declare no potential conflict of interest.

Received for publication: 27 February 2016.

Revision received: 30 April 2016.

Accepted for publication: 6 May 2016 .

This work is licensed under a Creative Commons Attribution 4.0 License (by-nc 4.0).

(C) Copyright T. Battista et al., 2016

Licensee PAGEPress, Italy

Emergency Care Journal 2016; 12:5854

doi:10.4081/ecj.2016.5854

two months, the stent is successfully removed without any complications.

\section{Discussion}

Spontaneous rupture of renal pelvis is an unusual event that is difficult to recognize because of its painful abdominal symptoms and the possibility of further complications due to peritoneal involvement and the development of a septic state. Its recognition is diagnostically challenging (taking into account that in literature a delayed diagnosis is reported in over $50 \%$ of cases $^{11}$ ) and may be life-saving.

Clinical presentation may be aspecific, often indistinguishable from a simple renal colic and the prognosis depends on underlying disease, degree of renal damage, location of rupture and infection occurrence. ${ }^{12}$

An accurate medical history is mandatory as well as a careful clinical examination. We want to stress the importance of the differential diagnosis in an emergency setting, because it is fundamental to treat patients with this condition as soon as possible.

In some cases symptoms mimic pain related to other abdominal pathogenesis, such as pyelonephritis, appendicitis, duodenal ulcer, biliary colic and cholecystitis.

Rupture of renal pelvis very often occurs in the setting of trauma, cancer, invasive or diagnostic procedures (the risk of extravasation during i.v. pyelogram is about $3 \%$ without any obstruction, while the risk increases from 5 to $33 \%$ when an obstruction is present) and also 
may be due to benign extrinsic compression of the ureter by a gravid uterus (it is rarely seen and is a significant complication in pregnancy). ${ }^{13}$ In absence of such conditions, spontaneous rupture at any level of urinary collecting system is generally due to an obstruction of urinary tract by calculus, ${ }^{12}$ with sudden increase of intraluminal pressure and this can cause rupture of its thin wall.
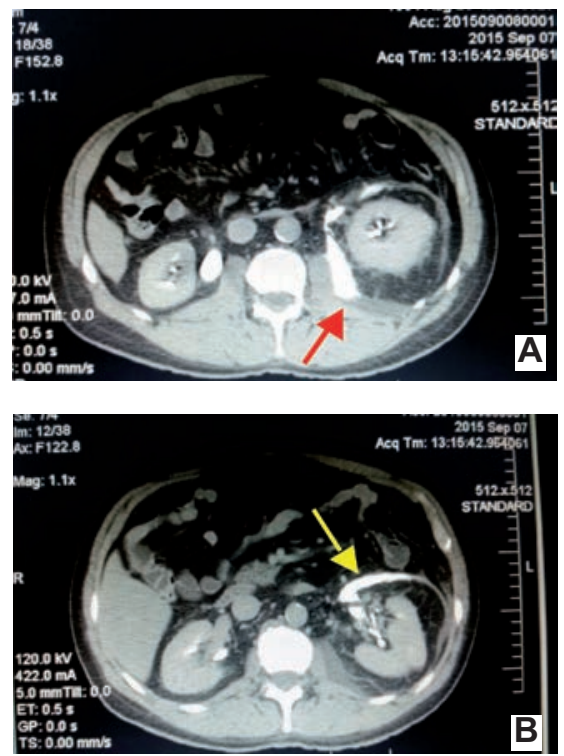

Figure 1. A contrast-enhanced computed tomography scan showing contrast leakage in perirenal and retroperitoneal spaces because of an ulceration of renal pelvis (arrows).

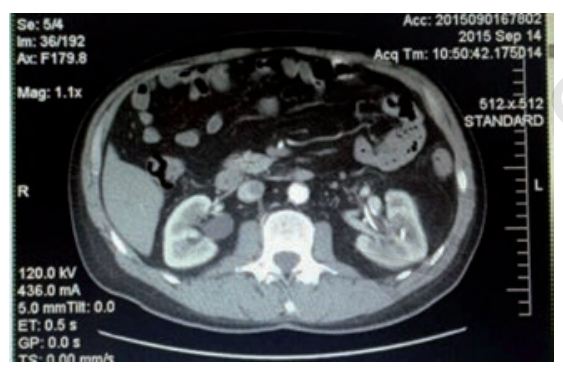

Figure 2. Computed tomography scan showing a good stent position, after which the patient can be discharged and advised to continue oral antibiotic therapy.
In our patient, absence of hydronephrosis on the ultrasound scan due to extravasation of urine in the perirenal space, observation of diverticula, peritoneal involvement and fever, at first confused the diagnosis, addressing it towards diverticulitis or other causes of acute abdomen or fever. In fact, clinical presentation in ER really was difficult to recognize because the renal symptoms were less intense while symptoms related to peritoneal involvement and incipient sepsis developed.

But, keeping in mind the most important initial clinical sign, that is typical renal calculotic pain complained of by patient at home and olyguria, the investigation continued with a contrast-enhanced CT making diagnosis clear. Subsequently, timely antibiotic and percutaneous interventional therapies have permitted pelvis detension and sepsis treatment.

In case of small ruptures a double $\mathrm{J}$ stent implantation or percutaneous nephrostomy is suitable; in the case of large ruptures a surgical intervention is mandatory. ${ }^{14}$ Dimensions of kidney calculus are important for their evolution: in fact small urinary stones $(<4 \mathrm{~mm})$ are ejected in 30-40 days; stones $>5 \mathrm{~mm}$ need an intervention (lithotripsy, ureteroscopy for ureterolithotomia) in over $50 \%$ of $\operatorname{cases}^{15}$ in a second step.

\section{Conclusions}

In conclusion, in a patient showing symptoms of renal colic with peritonitis syndrome and/or overlapping fever, one must keep in mind the possibility of a urinary tract rupture because a delayed diagnosis may be lifethreatening for the patient.

\section{References}

1. Paajanen H, Kettunen J, Tainio H, et al. Spontaneous peripelvic extravasation of urine as a cause of acute abdomen. Scand J Urol Nephrol 1993;27;333-6.

2. Koga S, Arakaki Y, Matsuoka M, et al. Spontaneous peripelvic extravasation of urine. Int Urol Nephrol 1992;24;465-9.
3. Spurlock JW, Burke TW, Dunn NP, et al. Calyceal rupture with perirenal urinoma in a patient with cervical carcinoma. Obset Gynecol 1987;70:511-3.

4. Buckley N, Smith JM. Renal extravasation of urine due to bladder outflow obstruction. J Urol 1984;132:1161-2.

5. Silverman SG, Leyendecker JR, Amis ES. What is the current role of CT urography and MR urography in the evaluation of the urinary tract? Radiology 2009;250:309-23.

6. Bonk JP, Basch RI, Cheris DN. Spontaneous rupture of the renal pelvis. Am J Roentgenol 1966;98:54-62.

7. Kpktener A, Unal D, Dilmen G, et al. Spontaneous rupture of renal pelvis caused by calculus: a case report. J Emer Med 2007;33:127-9.

8. Ashebu SD, Elshebiny YH, Dahniya MH. Spontaneous rupture of renal pelvis. Australas Radiol 2000;44:125-7.

9. Diaz ES, Buenrostro FG. Renal pelvis spontaneous rupture secondary to ureteral lithiasis: case report and bibliographic review. Arch Esp Urol 2011;64:640-2.

10. Ruchen CM, Menias CO, Bhalia S. Mimics of renal colic: alternative diagnoses at unenhanced helical CT. Radiographics 2004;24:511-28, 528-33.

11. Sierra Diaz E, Garcìa Buenrostro F. Renal pelvis spontaneous rupture secondary to ureteral lithiasis. Case report and bibliographic review. Arch Esp Urol 2011;64:6402.

12. Ferri E, Casoni GL, Morabito G, et al. Rupture of the renal pelvis complicating a renal colic: report of a case. Am J Emerg Med 2006;24:383-5.

13. Huang E, Sayegh R, Craigo S, Chelmow D. Rupture of the renal pelvis associated with intravenous fluid bolus. J Matern-Fetal Neonatal Med 2002;11:345-6.

14. Bogdanovic J, Djozic J, Idjuski S, et al. Succesful surgical reconstruction of ruptured renal pelvis following blunt abdominal trauma. Urol Int 2002;68:302-4.

15. Miller OF, Kane CJ. Time to stone passage for observed ureteral calculi: a guide for patient education. J Urology 1999;162:68891. 